\title{
LAS MONOCOTILEDÓNEAS ACUÁTICAS Y SUBACUÁTICAS DE MÉXICO
}

\author{
Antonio Lot \\ Departamento de Botánica, Instituto de Biología, \\ Universidad Nacional Autónoma de México \\ 04510 México, D.F., México \\ loth@ibiologia.unam.mx
}

\begin{abstract}
RESUMEN
Se presenta la revisión de la información sobre la riqueza y distribución de las monocotiledóneas acuáticas y subacuáticas (herbáceas) de México, con el fin de contribuir al mejor conocimiento de la flora de los humedales distribuidos en el territorio nacional. Se registraron 381 especies (392 taxa) pertenecientes a 104 géneros y 28 familias, referentes a las categorías de acuáticas estrictas y subacuáticas. Adicionalmente se documentaron 114 hidrófitas tolerantes o facultativas del hábitat palustre y lacustre. La mitad de las familias corresponden a taxa que la mayoría de los botánicos ubicarían como terrestres, pero con una representación significativa en el conjunto de plantas acuáticas mexicanas ( $71 \%$ de géneros y $75 \%$ de especies). El elemento endémico representa $14 \%$ del total de las monocotiledóneas. Su existencia en una gran variedad de hábitats y la representación en numerosos tipos de vegetación y comunidades, las colocan entre los elementos ecológicos y geográficos más diversos en el continente americano. En lo referente a las afinidades geográficas, encontramos que el componente meridional (46\%) supera por casi el doble al boreal (25\%), mientras que el elemento mexicano $(13.9 \%)$ es equivalente a la suma del cosmopolita y de las especies introducidas.

Palabras clave: distribución geográfica, hidrófitas, monocotiledóneas mexicanas, riqueza florística.
\end{abstract}

\section{ABSTRACT}

A review of the information on the richness and distribution of the Mexican aquatic and wetland (herbaceous) monocots, is presented, for the purpose of contributing to a 
better understanding of the flora distributed in the wetlands of the national territory. Three hundred eighty one species (392 taxa) belonging to 104 genera and 28 families, considered as strict aquatics or wetland plants, were registered; as well as 114 tolerant or facultative hydrophytes of lacustrine and pond habitats. Half of the 28 families correspond to taxa considered by most botanists as terrestrial, but they represent a large component of the Mexican aquatic flora ( $71 \%$ of the genera and $75 \%$ of the species). The endemics make up $14 \%$ of the monocots. Their presence throughout a great diversity of habitats and its representation in several vegetation types and communities, make them one on the most diverse ecological and geographical components in the American continent. About 46\% of the species belong to meridional elements, while $25 \%$ correspond to boreal one; while the Mexican element (13.9\%) is equivalent to the sum of the cosmopolitan and introduced species.

Key words: floristic richness, geographical distribution, hydrophytes, Mexican monocots.

\section{INTRODUCCIÓN}

Las llamadas universalmente "plantas acuáticas", también conocidas como macrófitas acuáticas o hidrófitas, corresponden a un conjunto de organismos vegetales que incluyen, en un sentido amplio, a las carofitas (Charophyta), musgos y hepáticas (Bryophyta), helechos y grupos afines (Pteridophyta), monocotiledóneas y dicotiledóneas (Magnoliophyta); sin embargo, los estudios florísticos de grandes regiones y países, se enfocan frecuentemente a las fanerógamas o a las plantas vasculares. El presente ensayo tiene como finalidad presentar un análisis preliminar de la riqueza de las monocotiledóneas acuáticas y subacuáticas (herbáceas) en la flora de México.

Cook (1996) al catalogar a las plantas acuáticas del mundo, registra 396 géneros de angiospermas de un total de 78 familias; de éstas, 44 son dicotiledóneas y 34 monocotiledóneas; la obra de Cook no incluye a la familia de las orquídeas, al considerar que ninguna de sus especies es una verdadera planta acuática. Los diferentes criterios aplicados en las floras de humedales de Norteamérica, en cuanto a la inclusión o no de especies subacuáticas, nos aleja de la posibilidad de analizar objetivamente la riqueza de las hidrófitas de manera comparativa entre regiones y países. Así tenemos que para la mayor parte de los Estados Unidos, en cuanto a las monocotiledóneas, se enumeran 152 géneros y 673 especies para el suroeste 
(Correll y Correll, 1972) y 175 géneros y 693 especies para el sureste (Godfrey y Wooten, 1979), en comparación con toda la región noreste de Norteamérica (Canadá y Estados Unidos) de mayor superficie y presencia de extensos humedales con un registro de 98 géneros y 618 especies (Crow y Hellquist, 2000). Es por ello, que algunos autores consideran conveniente analizar exclusivamente a las familias estrictamente acuáticas, como es el caso del estudio de Crow (1993), al comparar la diversidad de taxa entre el noreste de Norteamérica con México, que corresponden a una superficie equivalente; su análisis encuentra una relación de 45 géneros y 111 especies de México y de 37 y 126 para la parte de Norteamérica. Desafortunadamente la información sobre plantas acuáticas de Centro y Sudamérica es aún más incompleta, lo que no permite un análisis comparativo documentado (Crow, 1993).

El listado de Espejo y López-Ferrari (1993-2000) sobre las monocotiledóneas mexicanas, es un referente importante y punto de partida en el entendimiento de la participación de este conjunto de plantas, principalmente herbáceas, en la diversidad de la flora nacional. En dicha síntesis se enumeran cerca de 4,600 especies de 59 familias.

En la literatura existen algunas citas que ilustran particularmente el significado florístico de las monocotiledóneas acuáticas, como el de Ramírez García-Armora (1991) sobre los humedales del estado de Chiapas, encontrando $51 \%$ de géneros y $37 \%$ de especies con respecto a México. Otro caso contrastante por la ubicación geográfica y la gran diversidad de humedales temporales y permanentes es la península yucatanense (Campeche, Quintana Roo y Yucatán), para la que se registra dentro de las monocotiledóneas $60 \%$ de géneros y $48 \%$ de especies de hidrófitas estrictas (Gutiérrez, 2006). Las ciénagas de la cuenca alta del río Lerma en el estado de México son parte de las regiones hidrológicas prioritarias, con alta diversidad biológica y, como otros humedales, están amenazadas; su riqueza florística está representada por cerca de 100 hidrófitas de 65 géneros (Zepeda-Gómez et al., 2012). El estado de Tamaulipas es una de las entidades mejor conocidas en cuanto a la diversidad de la flora de los humedales; Mora-Olivo y Villaseñor (2007) registraron 426 especies pertenecientes a 213 géneros y 85 familias de plantas vasculares acuáticas y subacuáticas asociadas a humedales, que incluyen a los helechos y grupos aliados, angiospermas herbáceas y leñosas y una especie arbórea de gimnosperma. Considerando exclusivamente a las monocotiledóneas de familias acuáticas estrictas, México con respecto al mundo está representado por $42.3 \%$ de géneros y $16 \%$ de especies, al compartir 15 de las 19 familias con distribución mundial (Lot et al., 1998). Como antecedente directo al presente ensayo, se cuenta con una publicación 
sobre las afinidades florísticas de las monocotiledóneas acuáticas mesoamericanas (Lot y Novelo, 1992).

Algunos conceptos y consideraciones de las plantas acuáticas y de los humedales

Es importante precisar algunos de los conceptos que se emplean en el presente trabajo, que conforman la terminología acostumbrada en la generalidad de las publicaciones sobre aspectos florísticos y de la ecología de la vegetación de los humedales mexicanos. Los números de la riqueza florística, la representación taxonómica y la diversidad de los ambientes acuáticos, sin una definición acorde al contexto del trabajo publicado, causan confusión al lector, en particular al poco especializado en el tema, y principalmente por las múltiples interpretaciones de los términos y por la irremediable subjetividad en las clasificaciones.

El término "humedal" empieza a ser utilizado con mayor frecuencia en el medio académico hace cuatro décadas; su definición a un plano mundial continúa en el debate público a la fecha y, la aplicación del vocablo en diversas regiones del planeta, se limita a las grandes extensiones pantanosas, conocidas popularmente como lodazales y ciénagas. Por otro lado, es un concepto que intenta congregar la opinión oficial de la mayoría de los países en las estrategias de manejo de los recursos hídricos y en general de los ambientes acuáticos continentales y marinos costeros. Este punto favorece diversas interpretaciones del alcance del término hidrófita y en consecuencia, la metodología y la información, en muchos casos, no son comparables. En tal sentido es fundamental precisar qué se entiende por vegetación de humedales, que es mucho más amplia y compleja que las formaciones y comunidades de plantas acuáticas y subacuáticas. En el presente ensayo, la flora y vegetación de un humedal, incluye a formas de vida como las plantas epífitas, lianas, trepadoras, parásitas y, algunos arbustos y árboles que no corresponden a la vegetación acuática y subacuática estricta, pero que acompañan a las hidrófitas en diversas asociaciones y hábitats que quedan "atrapados" en muchos humedales, como por ejemplo los "petenes" de la Península de Yucatán.

En relación con el tema anterior, es importante revisar el concepto de hidrófita; afortunadamente cada día más estudiosos de los humedales han adoptado un sistema de clasificación, según Lot et al. $(1993,1998)$ basado en tres categorías: a) acuáticas estrictas, b) subacuáticas y c) tolerantes. Se fundamenta en el grado de dependencia de la planta al medio acuoso y en consecuencia está íntimamente relacionado al valor adaptativo de la especie a su hábitat. En este mismo sentido, otros autores como Tiner (1991) las agrupan en especies obligatorias y facultativas, dependiendo de la adaptación a la sumersión total o parcial (en tiempo y espacio) de 
la planta o de alguna de sus estructuras vegetativas o florales. Un concepto similar es ilustrado por Arber (1920) en la obra clásica Water plants: a study of aquatic angiosperms al señalar como "verdaderas plantas acuáticas" a diversos taxa del Orden Helobiae (Liliopsidae) representados por los géneros y especies que llevan a cabo el ciclo de vida completamente sumergidas.

\section{RIQUEZA FLORÍSTICA}

De acuerdo con los datos obtenidos, las monocotiledóneas acuáticas y subacuáticas documentadas para el territorio nacional suman un total de 392 taxa (381 especies y 11 taxa infraespecíficos), pertenecientes a 104 géneros y 28 familias (Cuadro 1); en una primera aproximación que contenga al conjunto de plantas consideradas tolerantes (114 especies), arrojaría un gran total de 500 monocotiledóneas acuáticas herbáceas (en las tres categorías). En este análisis quedaría pendiente documentar a las monocotiledóneas leñosas, representadas por las palmas y los bambúes de zonas inundables.

La mitad de las familias de monocotiledóneas registradas en este trabajo corresponden a taxa mayormente terrestres, pero con una representación significativa en el conjunto de plantas acuáticas mexicanas ( $71 \%$ de géneros y $75 \%$ de especies) al contribuir con 74 géneros y 287 especies de hábito acuático. Otro aspecto importante es el carácter endémico claramente superior entre los elementos de siete familias no acuáticas estrictas, con un valor de $94.3 \%$ (50 especies), con respecto a $0.5 \%$ (tres especies) de las familias con todos sus integrantes acuáticos. En el Cuadro 2 se agrupan las 12 familias con mayor riqueza para México entre las monocotiledóneas con representantes acuáticos; las ciperáceas y las gramíneas concentran a la gran mayoría de géneros (18 y 26 respectivamente) con 42\%, y de especies (125 y 75) con $52.4 \%$ del total (Diego-Pérez y González-Elizondo, en prensa); los elementos de ciperáceas y gramíneas de distribución restringida, representan $58.4 \%$, del total de endemismos registrados en las monocotiledóneas acuáticas de México.

\section{ENDEMISMO}

La participación con más de la mitad de endemismos en las Cyperaceae y Poaceae es notable, pero se deben considerar a otras familias, incluso más importantes cuantitativamente por la relación del número de géneros y especies y su can- 
Cuadro 1. Riqueza de la flora acuática herbácea monocotiledónea de México.

\begin{tabular}{lcccc}
\hline Familia & Géneros & Especies & $\begin{array}{c}\text { Especies } \\
\text { endémicas }\end{array}$ & $\begin{array}{c}\text { Categorías } \\
\text { infraespecíicas }\end{array}$ \\
\hline Alismataceae & 2 & 18 & 3 & 2 \\
Amaryllidaceae & 2 & 5 & 1 & \\
Araceae & 2 & 2 & - & \\
Bromeliaceae & 1 & 1 & - & \\
Cannaceae & 1 & 1 & - & \\
Commelinaceae & 5 & 7 & 2 & \\
Cymodoceaceae & 2 & 3 & - & \\
Cyperaceae & 18 & 125 & 21 & 5 \\
Eriocaulaceae & 4 & 16 & 6 & 1 \\
Hydrocharitaceae & 6 & 7 & - & 1 \\
Iridaceae & 3 & 15 & 6 & \\
Juncaceae & 1 & 18 & 1 & \\
Juncaginaceae & 2 & 2 & - & \\
Lemnaceae & 4 & 15 & - & \\
Limnocharitaceae & 2 & 9 & - & \\
Marantaceae & 1 & 1 & - & \\
Mayacaceae & 1 & 1 & - & \\
Najadaceae & 1 & 3 & - & \\
Orchidaceae & 9 & 15 & 3 & \\
Poaceae & 26 & 75 & 10 & \\
Pontederiaceae & 3 & 15 & - & \\
Potamogetonaceae & 2 & 10 & - & \\
Ruppiaceae & 1 & 2 & - & \\
Sparganiaceae & 1 & 2 & - & \\
Typhaceae & 1 & 2 & - & \\
Xyridaceae & 1 & 7 & - & \\
Zannichelliaceae & 1 & 1 & - & \\
Zosteraceae & 1 & 3 & - & \\
Total & 104 & 381 & 53 & \\
\hline & & & & \\
\hline
\end{tabular}

tidad de elementos mexicanos de distribución restringida. En el Cuadro 3 se puede apreciar, en parte, la desproporción del total de especies de las dos primeras familias con respecto al resto, pero si revisamos a las Eriocaulaceae e Iridaceae (Espejo y 
Cuadro 2. Familias (12) con el mayor número de especies de monocotiledóneas acuáticas en México.

\begin{tabular}{lcc}
\hline Familias & Géneros (\%) & Especies (\%) \\
\hline Cyperaceae & $18(17.3)$ & $125(32.8)$ \\
Poaceae & $26(25)$ & $75(19.7)$ \\
Alismataceae* & $2(0.2)$ & $18(4.7)$ \\
Eriocaulaceae & $4(0.4)$ & $16(4.2)$ \\
Lemnaceae* & $4(0.4)$ & $15(3.9)$ \\
Iridaceae & $3(0.3)$ & $15(3.9)$ \\
Pontederiaceae* & $3(0.3)$ & $15(3.9)$ \\
Orchidaceae & $9(8.0)$ & $15(3.9)$ \\
Potamogetonaceae* & $2(0.2)$ & $10(2.6)$ \\
Juncaceae & $1(0.1)$ & $18(4.7)$ \\
Hydrocharitaceae* & $6(0.6)$ & $7(1.8)$ \\
Commelinaceae & $5(0.5)$ & $7(1.8)$ \\
\hline
\end{tabular}

* Familias acuáticas estrictas.

López-Ferrari, en prensa), el porcentaje relativo de endemismos, considerando el número de especies por familia, es más alto; de las 10 especies de Eriocaulon, tres (E. capitulatum, E. jaliscanum y E. pringlei) son de distribución restringida, y cuatro (E. bilobatum, E. schiedeanum, E. schippii y E. seemannii) extienden su distribución a Centroamérica, por lo que siguiendo a Rzedowski (1998) se sumarían a la demarcación "Megaméxico II"; en el género Sisyrinchium con una decena de especies, cuatro ( $S$. cholewae, S. parvum, S. planicola y S. schaffneri) son endémicas a México, y dos (S. convolutum y S. scabrum) extienden su distribución a Centroamérica ("Megaméxico II"). Estos dos géneros son de hábitat palustre y la mayoría de las especies son subacuáticas, algunas de las cuales alcanzan los 3,000 m de altitud en pastizales y llanos anegados (Espejo y López-Ferrari, en prensa).

\section{DISTRIBUCIÓN Y AFINIDADES GEOGRÁFICAS}

Las monocotiledóneas presentan probablemente el espectro más amplio de distribución entre todos los elementos de la flora acuática mexicana. Su existencia en una gran variedad de hábitats y representación en numerosos tipos de vegetación 
Cuadro 3. Familias con elementos endémicos.

\begin{tabular}{lcc}
\hline Familias & $\begin{array}{c}\text { Número de } \\
\text { especies }\end{array}$ & Porcentaje \\
\hline Cyperaceae & 21 & $5.5 \%$ \\
Poaceae & 10 & $2.6 \%$ \\
Eriocaulaceae & 6 & $1.5 \%$ \\
Iridaceae & 6 & $1.5 \%$ \\
Alismataceae & 3 & $0.7 \%$ \\
Orchidaceae & 3 & $0.7 \%$ \\
Commelinaceae & 2 & $0.5 \%$ \\
Juncaceae & 1 & $0.2 \%$ \\
Amaryllidaceae & 1 & $0.2 \%$ \\
Total & 53 & $13.9 \%$ \\
\hline
\end{tabular}

y comunidades, las colocan entre los componentes ecológicos y geográficos más diversos en el continente americano. Las angiospermas marinas ilustran, por un lado, la evolución de formas de vida adaptadas a vivir en el fondo del océano a profundidades mayores de $30 \mathrm{~m}$, y por otro, existen monocotiledóneas acuáticas que crecen en condiciones extremas en páramos de altura por arriba de los 4,000 m s.n.m. En este sentido, solamente algunos grupos de criptógamas, como las algas, podrían competir con las monocotiledóneas acuáticas, en la colonización de ambientes de tan amplio intervalo altimétrico.

Al revisar de manera preliminar la distribución de los elementos de la flora en cuanto a su repartición geográfica (Cuadro 4), encontramos que el componente

Cuadro 4. Elementos geográficos de las monocotiledóneas acuáticas y subacuáticas de México. Porcentaje calculado con respecto al número total de especies.

\begin{tabular}{lcc}
\hline Elementos geográficos & $\begin{array}{c}\text { Número de } \\
\text { especies }\end{array}$ & Porcentaje \\
\hline Meridional & 176 & $46.1 \%$ \\
Boreal & 98 & $25.7 \%$ \\
Endémico a México & 53 & $13.9 \%$ \\
Cosmopolita (incluye Introducidas) & 54 & $14.2 \%$ \\
\hline
\end{tabular}


meridional (46\%) supera por casi el doble al boreal (25\%) en cuanto al número de especies, mientras que el elemento mexicano (13.9\%) es equivalente a la suma del cosmopolita y de las especies introducidas. Estas cifras confirman por un lado la predominancia meridional de la flora de México (Rzedowski, 1998) y por otro, precisan la generalización de que las plantas acuáticas son mayormente cosmopolitas.

\section{FORMAS DE VIDA, COMUNIDADES Y HÁBITAT}

El grueso de las monocotiledóneas acuáticas de México (325 especies) pertenecen a la categoría de hidrófita enraizada emergente (Fig. 1). Esta forma de vida es característica de la mayoría de las plantas acuáticas (estrictas, subacuáticas y tolerantes) e indicativa de la forma biológica predominante en los paisajes lacustres y palustres del país. El carácter de hierba emergente arraigada en sustratos sumergidos o anegados nos recuerda a las llamadas "plantas anfibias" y en consecuencia nos asocia con las terrestres que cohabitan en los límites de las zonas inundables. La desproporción tan alta de las hidrófitas enraizadas emergentes $(85.3 \%)$ con el resto



Fig. 1. Riqueza de las monocotiledóneas acuáticas y subacuáticas mexicanas por su forma de vida. hee= hidrófita enraizada emergente, hes=hidrófita enraizada sumergida, hef=hidrófita enraizada de hojas flotantes, hetp=hidrófita enraizada de tallos postrad, hls= hidrófita libre sumergida.Los números en los paréntesis corresponden a las especies. 
de las formas de vida, entre las cuales destacaría en segundo término las hidrófitas enraizadas sumergidas (8.1\%), refleja también la clara predominancia de plantas subacuáticas sobre las acuáticas estrictas.

En el Cuadro 5 se ilustran las principales comunidades y ambientes acuáticos y la participación proporcional de las monocotiledóneas en cada tipo, con la intención de dar una idea del conjunto de la flora acuática analizada con respecto a las grandes formaciones vegetales de México. Rzedowski (1998) estima que la vegetación acuática y subacuática de México representa 3\% con respecto al total de la flora, calculando en 1,000 el número de especies. Como se puede apreciar, las plantas acuáticas y subacuáticas herbáceas no sólo forman extensas comunidades y constituyen formaciones reconocidas en los tipos de vegetación de México (tulares y popales), sino que también se distribuyen en asociaciones que ocupan microhábitats en claros o pequeñas áreas discontinuas, en el interior de otros tipos de vegetación de regiones húmedas, pero también en regiones semiáridas. Estos elementos de la flora se encuentran pobremente representados en las colecciones herborizadas institucionales y con frecuencia pasan desapercibidos por los botánicos en las exploraciones de campo. Ejemplos poco conocidos en la literatura y

Cuadro 5. Distribución altitudinal y presencia de monocotiledóneas acuáticas en tipos de vegetación y comunidades bióticas.

\begin{tabular}{lccc}
\hline Tipo* & $\begin{array}{c}\text { Intervalo } \\
\text { altitudinal }(\mathrm{m})\end{array}$ & $\begin{array}{c}\text { Número de } \\
\text { especies }\end{array}$ & $\begin{array}{c}\text { Porcentaje con } \\
\text { respecto al total** }\end{array}$ \\
\hline Tular/popal/ciénaga & $0-2800$ & 79 & $20.7 \%$ \\
Bajo arrecifal & $(-) 0.5(-) 30$ & 6 & $1.5 \%$ \\
Intermareal rocoso & $0(-) 6$ & 3 & $0.7 \%$ \\
Manglar/marisma & $0-1$ & 20 & $5.2 \%$ \\
Sabana & $0-900$ & 18 & $4.7 \%$ \\
Matorral xerófilo & $0-2000$ & 17 & $4.4 \%$ \\
Bosque de coníferas & $2000-3000$ & 31 & $8.1 \%$ \\
Bosque tropical & $0-1500$ & 16 & $4.1 \%$ \\
caducifolio/subcaduc. & & & \\
Pastizal alpino & $2900-4000$ & 37 & $9.7 \%$ \\
\hline
\end{tabular}

* Algunas asociaciones ocupan microhábitats dentro de los tipos de vegetación terrestre incluidos.

** No se discriminan las especies exclusivas; el total corresponde a las 381 especies de monocotiledóneas. 
rara vez enlistados en las floras regionales son los elementos que se encuentran en los pastizales alpinos y ciénagas dentro del bosque de coníferas, con un intervalo altitudinal de 2,000 a 4,000 m: Carex boliviensis subsp. occidentalis, C. cochranei, C. durangensis, C. hermannii y C. rzedowskii (ciperáceas/todas endémicas mexicanas) (Diego-Pérez y González-Elizondo, en prensa); Eriocaulon microcephalum (Eriocaulaceae); Sisyrinchium convolutum, S. scabrum y S. tinctorium (Iridaceae) (Espejo y López-Ferrari, en prensa); Platanthera limosa, Schieedela durangensis y Spiranthes graminea (Orchidaceae) (Sosa y García, en prensa); Agrostis calderoniae, Muhlenbergia orophila y M. vaginata (Poaceae) (Herrera y Peterson, en prensa). En terrenos encharcados del matorral xerófilo pueden presentarse varias especies del género Juncus (Juncaceae), como J. acuminatus, J. liebmannii J. microcephalus y J. tenuis (Galván y Martínez, en prensa). En la sabana y zonas inundadas del bosque tropical: Eleocharis minima (hidrófita sumergida) y E. minutissima (Cyperaceae) (Diego-Pérez y González-Elizondo, en prensa); Paepalanthus lamarckii (Eriocaulaceae) (Espejo y López-Ferrari, en prensa); Xyris navicularis y X. smalliana (Xyridaceae) (Calderón de Rzedowski, en prensa).

\section{DISCUSIÓN}

El conjunto de monocotiledóneas acuáticas estrictas en el nivel de orden y de familia, es superior al de las dicotiledóneas ( $35 \%$ de las primeras contra $12 \%$ de las segundas); a nivel de género y especie, la participación aumenta a $66.6 \%$ y $73.2 \%$, contra $33.3 \%$ y $26.7 \%$. Sin embargo, al separar en el análisis al grupo de las plantas subacuáticas y a las formas leñosas, la situación se revierte, y aunque de momento no se puede documentar con la misma precisión que en las acuáticas estrictas, los cálculos iniciales basados en la participación del número de familias $(24.4 \%$ en monocotiledóneas $/ 75.5 \%$ en dicotiledóneas) y de géneros ( $25 \%$ monocotiledóneas $/ 74 \%$ dicotiledóneas), señala una tendencia aparentemente mayor en la riqueza florística de los ecosistemas acuáticos de México (datos fundados en la contribución de Lot et al., 1998).

La diversidad de las monocotiledóneas expresada en formas de vida, variedad de hábitat y microhábitat, formación de comunidades vegetales, endemismos y simetría en componentes provenientes del sur y del norte del continente, debe interpretarse como excepcional, y sin duda contribuye al significativo lugar que México tiene en el planeta en cuanto a la biodiversidad, particularmente en plantas vasculares de ambientes acuáticos. Esta primera mirada, basada en el conjunto de 
monocotiledóneas herbáceas acuáticas y subacuáticas, nos habla, en lo relativo a su fitogeografía, de que el territorio nacional es un corredor continuo sin fronteras políticas, en ambos sentidos con respecto al hemisferio sur y al hemisferio norte. La planicie inundable del Golfo de México, desde Tamaulipas hasta la península de Yucatán, lo ilustra (Lot, 2004).

Con mayor claridad se puede señalar que conforme profundizamos en el estudio botánico de las plantas acuáticas, sustentado en mayor exploración de campo y mejores colecciones con valor taxonómico, estaremos aproximándonos a un conocimiento cercano a la realidad de la diversidad de la Flora de México, antes de que la vulnerabilidad de las poblaciones vegetales y la fragmentación o modificación de los humedales sean irreversibles.

\section{AGRADECIMIENTOS}

El presente ensayo no hubiera sido posible sin el conjunto de datos proporcionados por diversos especialistas, a través de la colaboración que tuvieron en la realización de la obra "Plantas acuáticas mexicanas: una contribución a la flora de México", actualmente en revisión y próxima a publicarse. El autor de este trabajo (y editores de la obra, Rosalinda Medina Lemos y Fernando Chiang, todos del Instituto de Biología, Universidad Nacional Autónoma de México), agradecemos a los siguientes botánicos especialistas de las familias que integran el libro: Eduardo Domínguez-Licona (Alismataceae), Abisaí García-Mendoza (Amaryllidaceae), Leonardo Alvarado-Cárdenas (Araceae y Cannaceae), Adolfo Espejo y Ana Rosa López-Ferrari (Bromeliaceae, Commelinaceae, Eriocaulaceae e Iridaceae), Pedro Ramírez García-Armora (Cymodoceaceae, Ruppiaceae y Zosteraceae), Nelly Diego-Pérez y Socorro González-Elizondo (Cyperaceae), Martha Olvera García (Hydrocharitaceae, Lemnaceae, Limnocharitaceae, Pontederiaceae y Zannichelliaceae), Raquel Galván Villanueva y Clara Martínez Domínguez (Juncaceae), Gabriel Sánchez Ken (Juncaginaceae), Arturo Mora Olivo y Jorge Luis Mora López (Najadaceae), Maite Lascurain (Marantaceae), Rosalinda Medina Lemos (Mayacaceae), Victoria Sosa y Javier García (Orchidaceae), Yolanda Herrera y Paul M. Peterson (Poaceae), Mahinda Martínez (Potamogetonaceae), Carmen Zepeda (Typhaceae) y Graciela Calderón de Rzedowski (Xyridaceae).

De manera especial agradezco a Rosalinda Medina Lemos por la revisión de este trabajo y por la consistente ayuda brindada en todo el proceso de organización de la información taxonómica. A Lorena Villanueva-Almanza por la traducción al inglés del resumen. 
La presente contribución está dedicada a la memoria de Raymundo García Alcántara y Armando Butanda Cervera, personas que a lo largo de sus vidas, consagraron toda su capacidad y esfuerzo a la compilación de literatura botánica, preferentemente con relación a la historia, y a la asistencia como bibliotecario especializado (Raymundo) y Técnico en Biblioteconomía (Armando), del Instituto de Biología de la Universidad Nacional Autónoma de México.

\section{LITERATURA CITADA}

Arber, A. 1920 (1972). Water plants, a study of aquatic angiosperms. J. Cramer. New York, USA. $436 \mathrm{pp}$.

Calderón de Rzedowski, G. en prensa. Xyridaceae. In: Lot, A., R. Medina-Lemos y F. Chiang (eds.). Plantas acuáticas mexicanas: una contribución a la Flora de México. Universidad Nacional Autónoma de México. México, D.F., México.

Cook, C. D. K. 1996. Aquatic plants book. SPB Academic Publications. Amsterdam, The Netherlands. 228 pp.

Correll, D. S. y H. B. Correll 1972. Aquatic and wetland plants of Southwestern United States. Enviroment Protection Agency. Washington, D.C., USA. 1777 pp.

Crow, G. E. 1993. Species diversity in aquatic angiosperms: latitudinal patterns. Aq. Bot. 44: 229-258.

Crow, G. E. y C. B. Hellquist. 2000. Aquatic and wetland plants of Northeastern North America. Vol. 2. Angiosperms: monocotyledons. The University of Wisconsin Press. Wisconsin, USA. 400 pp.

Diego-Pérez, N. y S. González-Elizondo. en prensa. Cyperaceae. In: Lot, A., R. MedinaLemos y F. Chiang (eds.). Plantas acuáticas mexicanas: una contribución a la Flora de México. Universidad Nacional Autónoma de México. México, D.F., México.

Espejo, A. S. y A. R. López-Ferrari. 1993-2000. Las monocotiledóneas mexicanas, una sinopsis florística, 1. Lista de referencia. Partes I a XI. Consejo Nacional de la Flora de México, A.C., Universidad Autónoma Metropolitana Iztapalapa, Comisión Nacional para el Conocimiento y Uso de la Biodiversidad. México, D.F., México. 337 pp.

Espejo, A. S. y A. R. López-Ferrari. en prensa. Eriocaulaceae e Iridaceae. In: Lot, A., R. Medina-Lemos y F. Chiang (eds.). Plantas acuáticas mexicanas: una contribución a la Flora de México. Universidad Nacional Autónoma de México. México, D.F., México.

Galván, R. V. y C. E. D. Martínez. en prensa. Juncaceae. In: Lot, A., R. Medina-Lemos y F. Chiang (eds.). Plantas acuáticas mexicanas: una contribución a la Flora de México. Universidad Nacional Autónoma de México. México, D.F., México.

Godfrey, R. K. y J. W. Wooten. 1979. Aquatic and wetland plants of Southeastern United States: Monocotyledons. The University of Georgia Press. Athens, Georgia, USA. 712 pp.

Gutiérrez, B. C. 2006. Lista de plantas acuáticas vasculares de la Península de Yucatán, México. Polibotánica 21: 75-87. 
Herrera, Y. y P. M. Peterson. en prensa. Poaceae. In: Lot, A., R. Medina-Lemos y F. Chiang (eds.). Plantas acuáticas mexicanas: una contribución a la Flora de México. Universidad Nacional Autónoma de México. México, D.F., México.

Lot, A. 2004. Flora y vegetación de los humedales de agua dulce en la zona costera del Golfo de México. In: Caso, M., I. Pisanty y E. Ezcurra (comp.). Diagnóstico ambiental del Golfo de México. Secretaría del Medio Ambiente y Recursos Naturales, Instituto Nacional de Ecología, Instituto de Ecología, A.C., Harte Research Institute for Gulf of Mexico Studies. México, D.F., México. pp. 521-553.

Lot, A. y A. Novelo. 1992. Afinidades florísticas de las monocotiledóneas acuáticas mesoamericanas. In: Darwin, S. P. y A. L. Welden (eds.). Biogeography of Mesoamerica. Tulane Stud. in Zool. and Bot. (Supplementary Publication) 1: 147-153.

Lot, A., A. Novelo y P. Ramírez. 1993. Diversity of Mexican aquatic vascular plant flora. In: Ramamoorthy, T. P., R. Bye, A. Lot y J. Fa (eds.). Biological Diversity of Mexico: origins and distributions. Oxford University Press. New York, USA. pp. 577-594.

Lot, A., A. Novelo y P. Ramírez. 1998. Diversidad de la flora acuática mexicana. In: Ramamoorthy, T. P., R. Bye, A. Lot y J. Fa (comp.). Diversidad biológica de México: orígenes y distribución. Universidad Nacional Autónoma de México. México, D.F., México. pp. 563-578.

Mora-Olivo, A. y J. L. Villaseñor. 2007. Diversidad y distribución de la flora vascular acuática de Tamaulipas, México. J. Bot. Res. Inst. Texas 1(1): 511-527.

Ramírez García-Armora, J. P. 1991. Flora vascular (monocotiledóneas) del Estado de Chiapas. Tesis de Maestría. Facultad de Ciencias, Universidad Nacional Autónoma de México. México, D.F., México. 113 pp.

Rzedowski, J. 1998. Diversidad y orígenes de la flora fanerogámica de México. In: Ramamoorthy, T. P., R. Bye, A. Lot y J. Fa (comp.). Diversidad biológica de México: orígenes y distribución. Universidad Nacional Autónoma de México. México, D.F., México. pp. 129-145.

Sosa, V. y J. García. en prensa. Orchidaceae. In: Lot, A., R. Medina-Lemos y F. Chiang (eds.). Plantas acuáticas mexicanas: una contribución a la Flora de México. Universidad Nacional Autónoma de México. México, D.F., México.

Tiner, R. 1991. The concept of a hydrophyte for wetland identification. BioScience 41: 236247.

Zepeda-Gómez, C., A. Lot-Helgueras, X. N. Antonio y D. Madrigal-Uribe. 2012. Florística y diversidad de las ciénegas del río Lerma, Estado de México, México. Acta Bot. Mex. 98: 23-49. 